\title{
SYNTHESIS AND CHARACTERIZATION OF PURE ZINC OXIDE NANOPARTICLES AND NICKEL DOPED ZINC OXIDE NANOPARTICLES
}

\author{
J.S. Mayekar ${ }^{1}$, V.S. Dhar ${ }^{2}$, S. Radha $^{3}$ \\ ${ }^{1}$ Assistant Professor, Department of Physics, Jai Hind College, Mumbai, Maharashtra, India \\ ${ }^{2}$ Associate Professor, V.S. Dhar, Department of Physics, Jai Hind College, Mumbai, Maharashtra, India \\ ${ }^{3}$ Associate Professor, Department of Physics, University of Mumbai, Maharashtra, India.
}

\begin{abstract}
In this paper, Zinc oxide nanoparticles are synthesized by simple wet chemical precipitation method. Zinc nitrate and sodium hydroxide are used as the starting materials.Zinc oxide nanoparticles are formed at a very low temperature of the order of $80^{\circ} \mathrm{C}$. Nickel doped zinc oxide nanoparticles are synthesized in two steps. In first step precipitate is obtained by reduction of mixture of zinc nitrate, ferric nitrate and starch by sodium hydroxide solution while in second step the given precipitate is thermally decomposed at high temperature of the order of $400^{\circ} \mathrm{C}$. The crystallinity of the synthesized nanoparticles is then confirmed by $X$ ray diffraction spectroscopy (XRD).The elemental composition of the powder is detected by Energy Dispersive X ray spectroscopy (EDAX). The morphology of the powder is investigated by Scanning Electron Microscopy (SEM). Magnetic characterization of nickel doped zinc oxide nanoparticles is done by Squid Magnetometer. Low temperature magnetization behavior revealed ferromagnetic behavior of sample.
\end{abstract}

Key Words: Zinc oxide nanoparticles, Nickel doped ZnO, Antibacterial activity, Squid magnetometer, SEM

\section{INTRODUCTION}

Zinc Oxide $(\mathrm{ZnO})$ is a unique material with a direct band gap (3.37eV) and large exciton binding energy of $60 \mathrm{MeV}$. Because of its exceptional optical and electrical properties, zinc oxide has been extensively used in many technological applications such as thin film transistors [1], gas sensors [2], transparent conductor [3], Bio medical [4] and piezoelectric application [5].Various methods have been employed by researchers for synthesis of $\mathrm{ZnO}$ nano structured materials like nonionic polymer assisted thermolysis [6], conventional solid state reaction [7], sol-gel method [8], electron beam deposition [9], an electro chemical route [10], chemical coprecipitation method [11] etc. Among these methods, chemical co-precipitation method is the best one, because it is simple, less expensive and has high yield rate.

II-VI semiconductor materials at nanometer scale plays key role in various applications due to their great potential. $\mathrm{ZnO}$ is an important II-VI semiconductor having roomtemperature (RT) ferromagnetism (FM) when doped with transition metals (TM) $[12,13,14,15]$. This makes $\mathrm{ZnO}$ one of the most promising materials for potential applications in spintronics and as diluted magnetic semiconductor (DMS) material. The aim of this study is the enhancement of magnetic properties of the $\mathrm{ZnO}$ nanoparticles by doping. The main challenge for this kind of materials is to attain their magnetic characterization at room temperature in order to be useful for technological applications. In the present study, the room temperature ferromagnetic nano particles are synthesized by chemical co-precipitation method. Structural, morphological and magnetic properties of the synthesized samples are investigated.

\section{EXPERIMENTAL}

Zinc oxide nanoparticles are synthesized by precipitation method [11]. For the synthesis of nickel doped zinc oxide nanoparticles, $14.87 \mathrm{gm}$ of zinc nitrate and $0.1 \mathrm{gm}$ of starch is dissolved in $100 \mathrm{ml}$ of distilled water. This solution is heated till the temperature reaches $60^{\circ} \mathrm{C}$. Add $1 \mathrm{M}$ of sodium hydroxide solution to above solution drop by drop with continuous stirring by magnetic stirring. Prepare another solution of $2.908 \mathrm{gm}$ of nickel nitrate and $0.1 \mathrm{gm}$ of starch in $100 \mathrm{ml}$ of distilled water. Add this solution to above solution and stir for two hours using magnetic stirrer. Keep the solution overnight. Precipitate is formed. Oven dry the precipitate at $100^{\circ} \mathrm{C}$. Powder is formed. Keep this powder in Muffel furnace for $6 \mathrm{hrs}$ at $400^{\circ} \mathrm{C}$. Nickel doped zinc oxide nanoparticles are formed.

\section{RESULTS AND DISCUSSIONS}

\subsection{Ray Diffraction Spectroscopy}

The X-ray diffraction (XRD) patterns of the powdered samples were recorded using an Xpert PRO diffractometer with $\mathrm{Cu} K \alpha$ radiation at room temperature. The crystallite size was estimated using the Scherrer equation from the full width at half maximum of the major XRD peak. 

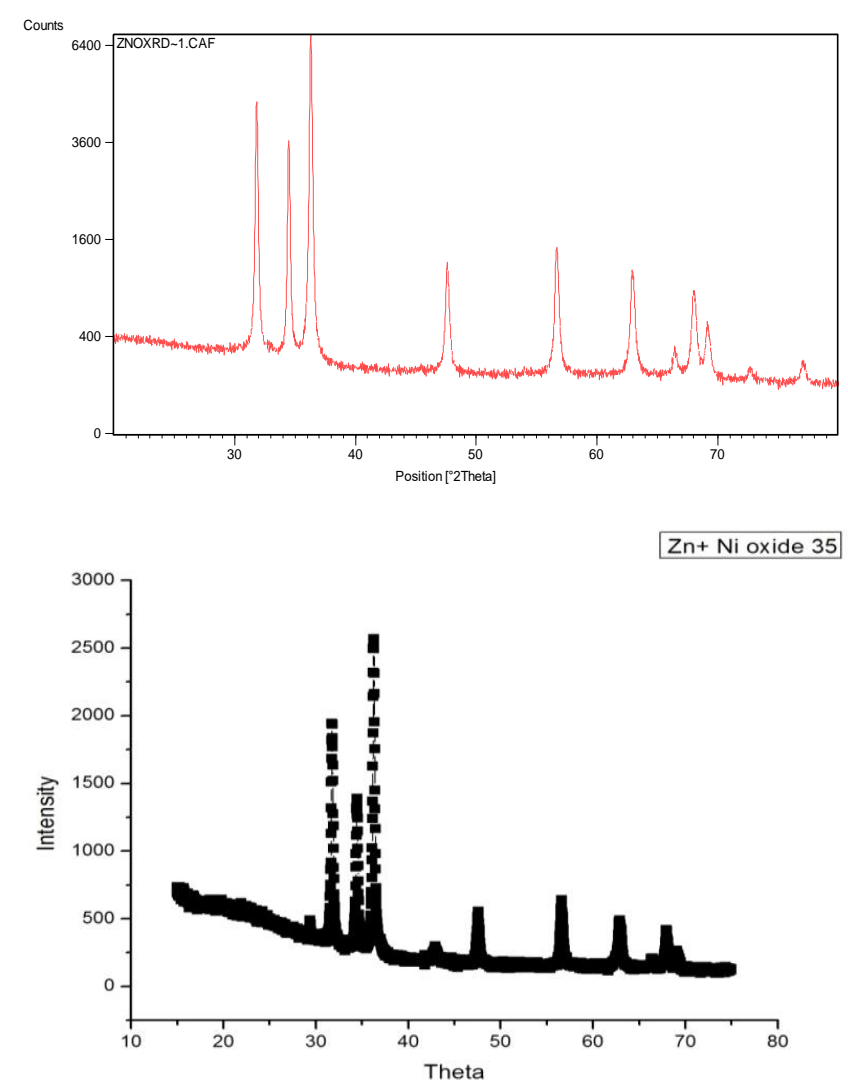

Fig 1 and 2: $X$ ray diffraction patterns of Zinc oxide and nickel doped zinc oxide nanoparticles

The crystalline structure was analyzed by X-ray diffraction (XRD) using $\mathrm{CuK} \alpha$ radiation $(1.5405 \AA)$ diffractometer operating at $40 \mathrm{kV}, 30 \mathrm{~mA}$ for angles between $2 \theta=10^{\circ}$ and $80^{\circ}$ in $0.02^{\circ}$ steps. The sharp and intense peaks indicate that the samples are highly crystalline. The XRD peaks for (100), (002), (101), (102), (110), (103) and (112) planes indicates the formation of phase pure wurtzite structure of $\mathrm{ZnO}$.

Lattice constants ' $a$ ' and ' $c$ ' are calculated from the XRD data and shown in Table 1. It shows good agreement with the standard value $(\mathrm{a}=\mathrm{b}=3.249 \AA, \mathrm{c}=5.206 \AA)$ [JCPDS-361451].

No additional peaks corresponding to the secondary phases of nickel oxide were obtained. We can conclude that the wurtzite structure of $\mathrm{ZnO}$ is not changed by the $\mathrm{Ni}$ substitution and that $\mathrm{Ni}^{2+}$ occupies the $\mathrm{Zn}^{2+}$ site into the crystal lattice.Table 1 shows that the lattice constants of Zn1-xNixO ( $x=0.055)$ are slightly smaller than those of pure $\mathrm{ZnO}$, because of the difference between the ionic radius of the elements $\left[\mathrm{r}\left(\mathrm{Zn}^{2+}\right)=0.60 \AA\right.$ and $\mathrm{r}\left(\mathrm{Ni}^{2+}\right)=$ $0.55 \AA ̊ A]$.

\subsection{Energy Dispersive $X$ ray Spectroscopy}

The energy dispersive X-ray analysis of Pure Zinc oxide nanoparticles and Nickel doped Zinc Oxide nanoparticles are shown in the Figs 3 and 4. It is evident from the X-ray patterns that all the dopants are found in the respective spectrum. In addition to that interestingly it is observed there are no foreign materials present in the spectrum. It is an added confirmation for the purity of the samples. Quantitative analysis of all the samples is put down in the tables 2.. Also in the table it is seen that no impurities are found out.

Table -1: Lattice parameters of Pure $\mathrm{ZnO}$ and Nickel doped

\begin{tabular}{|l|l|l|}
\hline Lattice constant & Pure Zinc Oxide & $\begin{array}{l}\text { Nickel doped } \\
\text { zinc oxide }\end{array}$ \\
\hline $\mathrm{a}\left(\mathrm{A}^{0}\right)$ & 3.250 & 3.248 \\
\hline $\mathrm{c}\left(\mathrm{A}^{0}\right)$ & 5.209 & 5.208 \\
\hline
\end{tabular}
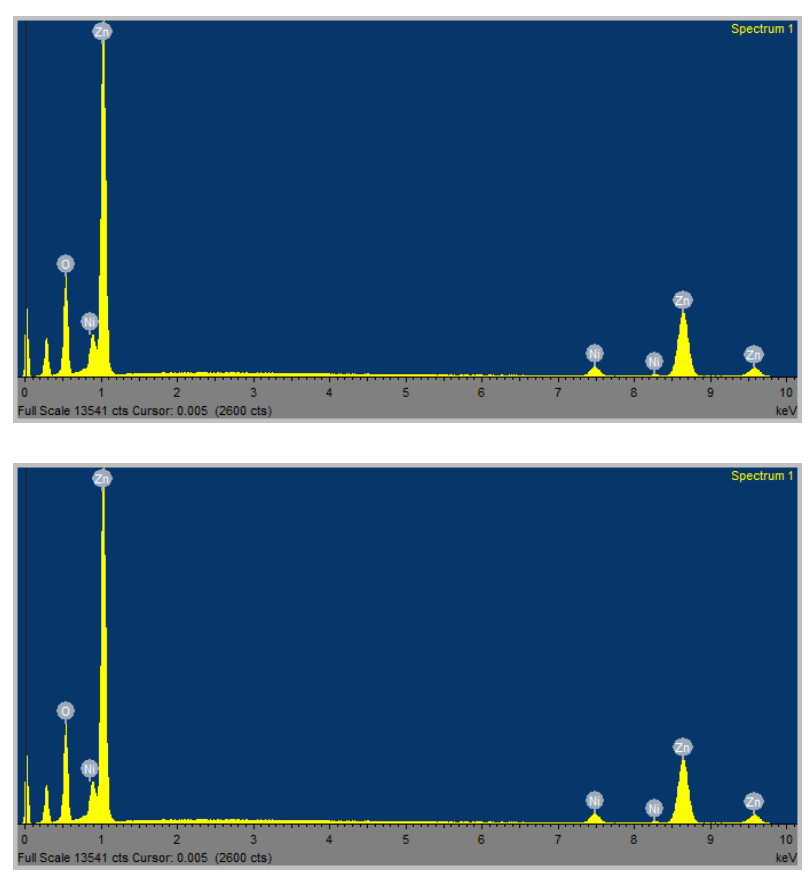

Fig 3 and 4: EDAX spectrum of Pure and Nickel doped $\mathrm{ZnO}$ nanoparticles

Table 2: Elemental Compositionof doped and undoped Zinc oxide nanoparticles

\begin{tabular}{|l|l|l|l|}
\hline Spectrum & $\mathrm{Zn}$ & $\mathrm{O}$ & $\mathrm{Ni}$ \\
\hline $\mathrm{ZnO}$ & 67.96 & 31.84 & ----- \\
\hline Nickel doped $\mathrm{ZnO}$ & 36.57 & 60.88 & 2.56 \\
\hline
\end{tabular}

\subsection{Scanning Electron Microscopy}

SEM images shows that the zinc oxide nanoparticles have flower like structures while nickel doped zinc oxide nanoparticles are spherical in nature.The zinc oxide flower like structures have $20 \mathrm{~nm}$ diameter and $200 \mathrm{~nm}$ length while the synthesized nickel doped ZnO NPs are having 50 $\mathrm{nm}$ size.These sizes match with that of calculated by Debye Scherrer formula. 


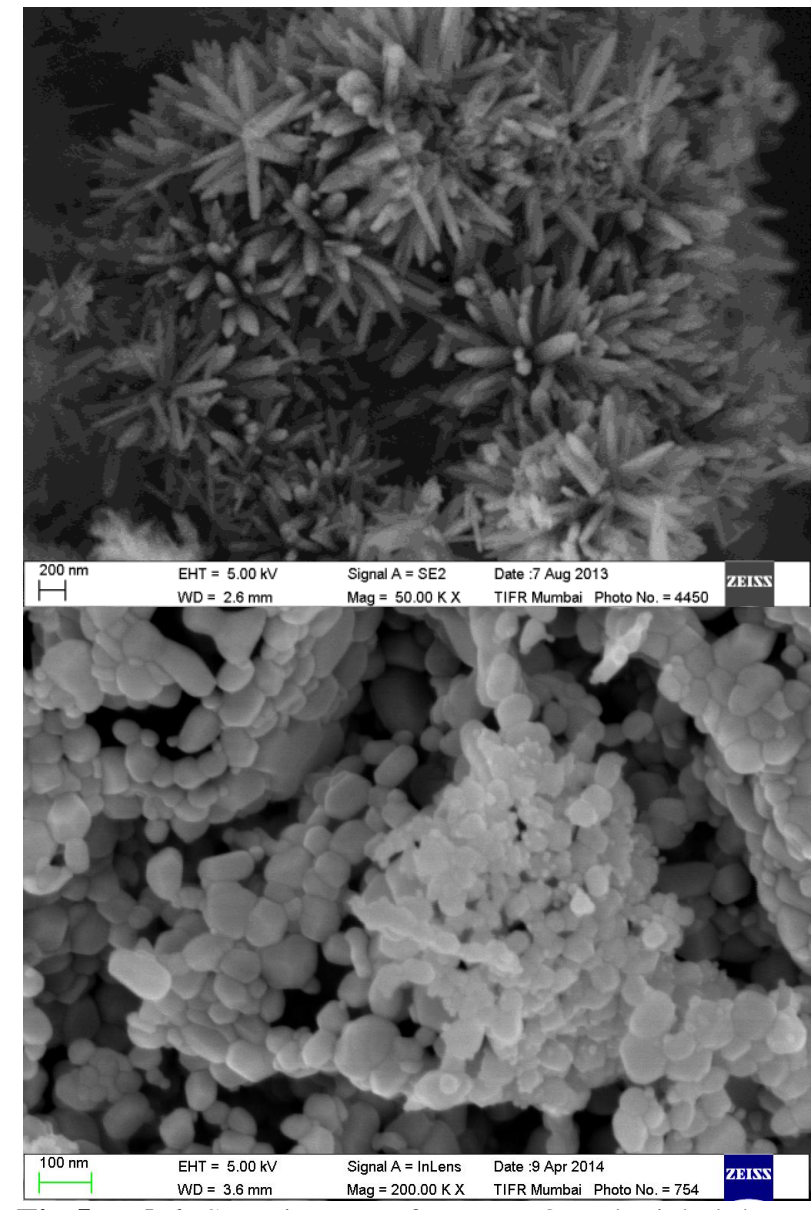

Fig 5 and 6: SEM images of Pure $\mathrm{ZnO}$ and Nickel doped $\mathrm{ZnO}$ NPs

\subsection{Ferromagnetism In Nickel Doped Zinc Oxide}

\section{Nanoparticles}

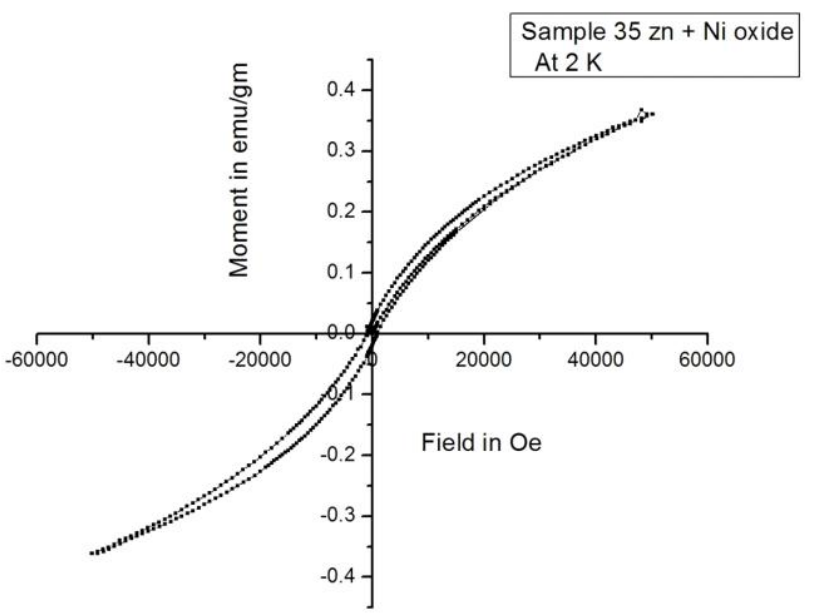

Fig 7: $\mathrm{BH}$ loop of nickel doped $\mathrm{ZnO} \mathrm{Nps}$

The magnetic characterization is done on Squid magnetometer. The BH loop study shows that the sample shows ferromagnetic behaviour. The saturation magnetization is found to be of the order of $0.37 \mathrm{emu} / \mathrm{gm}$.

\section{CONCLUSIONS}

The ferromagnetism is observed due to the presence of defect related mechanism such as oxygen vacancies.The Nickel doping induces ferromagnetic behaviour in the Zinc oxide nanoparticles. The $\mathrm{Ni}$ doped $\mathrm{ZnO}$ nanoparticles of the present work having low magnetization could form the diluted magnetic semiconductors for spintronic applications.

\section{ACKNOWLEDGEMENT}

The authors acknowledge Tata Institute of Fundamental Research, Mumbai for Characterization techniques.

\section{REFERENCES}

[1] K. Nomura, H. Ohta, K. Ueda, T. Kamiya, M. Hirano, H. Hosono, Thin film transistor fabricated in single crystalline transparent oxide semiconductor, Science, 300, 1269 (2003)

[2] S.Roy,S.Basu, Improved zinc oxide film for gas sensor application,Bull. Mater. Sci., Vol. 25, No. 6, November 2002, pp. 513-515.

[3] Takashi ogi, Darmawan Hidayat, Ferry Iskandar,Agus Purwanto,Kikuo Okuvama, Direct Synthesis of highly crystalline transparent conducting oxide nanoparticles by low pressure spray pyrolysis, Advanced Powder Technology, Volume 20 Issue 2, March 2009,203-209.

[4] Zhang Y., Nayak TR, Hong H, Cai W Biomedical applications of zinc oxide nanomaterials, Curr. Mol. Med.2013 dec, 13(10),1633-45

[5] Min- Hua Zhao, Zhong-Lin Wang, Scott X. Mao,Piezoelectric characterization of individual zinc oxide nanobelt probed by piezoresponse force microscope, Nano Lett., 2004, 4(4), pp 587-590

[6] Joshua W. Kriesel, Melissa S. Sander, T. Don Tilley, Block copolymer -Assisted synthesis of mesoporous ,Multicomponent oxides by nonhydrolytic,thermolytic decomposition of molecular precursors in Nonpolar media,Chem Mater. 2001,13(10), pp 3554-3563

[7] Zhi-Peng Sun Lang Liu, Li Zhang and Dian- Zeng Jia, Rapid Synthesis of $\mathrm{ZnO}$ nano-rods by one -step, roomtemperature, Solid-state reaction and their gas-sensing properties, Nanotechnology, 2006 17, 2266

[8] Lubomir Spnahel, Marc A.Anderson, Semiconductor clusters in the sol-gel process: quantized aggregation,gelation and crystal growth in concentrated zinc oxide colloids, J.am. Chem. Soc., 1991, 113(8),pp 2826-2833

[9] Shunichi hayamizu, Hitoshi Tabata, Hidekazu Tanaka, Tomoji Kawai, Preparation of crystallized zinc oxide films on amorphous glass substrates by pulsed laser deposition, J. Appl. Phys., 80, 787(1996)

[10] Shailaja Mahamuni, Kavita Borgohain, B.S.Bendre, Valerie L.Leppert, Subhash H. Risbud, Spectroscopic 
and structural characterization of electrochemically green $\mathrm{ZnO}$ quantum dots, J.Appl. Phys., 85, 2861

[11] Mayekar Jyoti, Dhar Vijay, S. Radha,To study the role of temperature and sodium hydroxide concentration in the synthesis of zinc oxide nanoparticles,International Journal of Scientific and Research Publications,2013, vol. 3, Issue 11.

[12]M. Venkatesan, C. B. Fitzgerald, J.G. Lunney, J.M.D.Coey, Anisotropic ferromagnetism in substituted zinc oxide,Phy. Rev.Lett, 2004, 93, 177206

[13] K.Sato,H.Katayama-Yoshida,Electronic structure and ferromagnetism of transition-metal-impurity-doped zinc oxide, Physica B: Condensed Matter, 2001, vol. 308310, pp 904-907

[14] Kazunori Sato, Hiroshi katayama-Yoshida, Material design for transparent ferromagnets with $\mathrm{ZnO}$-based magnetic semiconductors, Applied Physics, volume 39, Part 2, Number 6B

[15] S. Risbud, N.A.Spaldin, Z.Q.Chen, S.Stemmer, Ram Seshadri, magnetism in Polycrystalline cobaltsubstituted zinc oxide, Phys. Rev. B, 2003 68, 205202 